University of Nebraska - Lincoln

DigitalCommons@University of Nebraska - Lincoln

Faculty Publications in Computer \& Electronics Electrical \& Computer Engineering, Department Engineering (to 2015)

1999

\title{
DS CDMA Scheme for WATM with Errors and Erasures Decoding
}

\author{
Beata J. Wysocki \\ University of Nebraska-Lincoln \\ Hans-Jürgen Zepernick \\ Curtin University of Technology \\ Tadeusz Wysocki \\ University of Nebraska-Lincoln, wysocki@uow.edu.au
}

Follow this and additional works at: https://digitalcommons.unl.edu/computerelectronicfacpub

Part of the Computer Engineering Commons

Wysocki, Beata J.; Zepernick, Hans-Jürgen; and Wysocki, Tadeusz, "DS CDMA Scheme for WATM with Errors and Erasures Decoding" (1999). Faculty Publications in Computer \& Electronics Engineering (to 2015). 36.

https://digitalcommons.unl.edu/computerelectronicfacpub/36

This Article is brought to you for free and open access by the Electrical \& Computer Engineering, Department of at DigitalCommons@University of Nebraska - Lincoln. It has been accepted for inclusion in Faculty Publications in Computer \& Electronics Engineering (to 2015) by an authorized administrator of DigitalCommons@University of Nebraska - Lincoln. 


\title{
DS CDMA Scheme for WATM with Errors and Erasures Decoding
}

\author{
Beata J. Wysocki*, Hans-Jürgen Zepernick*, and Tadeusz A. Wysocki** \\ *Australian Telecommunications Research Institute \\ Curtin University of Technology \\ GPO Box U 1987, Perth WA 6845, Australia \\ E-mail: \{beata, hans\}@atri.curtin.edu.au \\ ** University of Wollongong \\ School of Electrical, Computer and Telecommunications Engineering \\ Northfields Avenue, Wollongong NSW 2522, Australia \\ E-mail:wysocki@uow.edu.au
}

\begin{abstract}
In the paper, we present simulation results for the 13 channel DS CDMA WATM LAN utilising optimized complex spreading signatures based on Walsh functions. The method to obtain those optimized spreading signatures, as well as the full set of the coefficients giving the minimum level of cross-correlation between any pair of the channels is shown. The resultant system BER as well as the distribution of errors within WATM cells is given. The obtained results indicate that with the application of a hybrid ARQ scheme with errors and erasures decoding, the number of WATM cells which would require retransmission is in the order of $0.46 \%$.
\end{abstract}

\section{INTRODUCTION}

During the 1990s, direct sequence spread spectrum code division multiple access (DS CDMA) technology [1] has matured as a technique to provide multiple access to the radio channel for mobile communications. For example, it is used in mobile telephony compliant with IS-95 standard [2]. Recently, it has gained even more attention with ETSI approving it as a technology for the European third generation mobile system (UMTS) [3]. It is expected that wireless LANs will complement the third generation mobile telephony, providing a wireless vehicle for high rate multimedia applications.

ATM (Asynchronous Transfer Mode) is the technique being the commonly accepted standard for the broadband networks, and is also becoming accepted as a common nominator for all types of services and networks. Also, there has been widespread use of wireless communications to support users' requirements for wireless access and terminal mobility in such scenarios as cellular telephony networks and narrowband wireless LANs. As a further advancement, users are now beginning to require broadband services and terminal mobility to be provided together through wireless access to ATM networks.

The major benefits of many DS CDMA is that it can be effective in combating problems related to multi-path propagation, while providing good interference from other, narrowband devices operating in the same frequency band. This is, however. dramatically reduced if only a small processing gain [1] can be achieved, as in the case of WATM LANs where the ratio of available bandwidth to the proposed data rate is in the order of less than 20 (e.g. 2.4 $\mathrm{GHz}$ ISM band). Under such conditions, in-band jammers, like other channels of the same WLAN acquired by means of CDMA cause severe multiple access interference (MAI), which may block the communication.

In theory, cancellation of that type of interference is possible if each of the users utilise orthogonal signals to transmit the data [1]. If delays between transmitters and receiver are anyhow different, as is generally the case of terminal to base station (BS) transmission, the signals received by the $\mathrm{BS}$ cannot be regarded as orthogonal Within the $50 \mathrm{~m}$ coverage area those differences may be in the order of a few spreading code symbols (chips) depending on the data rate. This effect is particularly critical for very short spreading sequences, like 16-bit Walsh functions.

In [4] we have proposed the method to reduce the ISI and MAI for DS CDMA wireless networks, and in [5], we optimized the scheme for the system using 13 spreading signatures based on the 16-bit Walsh functions. This paper deals with further design of DS CDMA WATM LAN where an error control mechanism is employed.

The paper is organized as follows. In Section II, we briefly discuss the method of designing the complex multilevel spreading signatures based on 16-bit Walsh functions. Section III presents results of simulated error performance for the 13 channel ATM WLAN utilizing the complex multilevel spreading signatures. In Section IV, we describe the error control scheme with hard decision optimized for our DS CDMA system. Section V then extends the concept to errors and erasures decoding. Section VI concludes the paper.

\section{DESIGN METHOD}

In $[4]$, we have described the method to reduce ISI and MAI for non-synchronized CDMA signals by means of a modification to the carrier waveform. The modified carrier 
has been obtained by a regular distortion to the frequency of the original carrier, resulting in the ith user line signal $s_{i}(t)$ expressed by:

$$
s_{i}(t)=g_{i}(t) \cdot \cos \left[\omega_{c} t+\int_{0}^{t} w_{i}(\tau) d \tau+\phi_{i}(t)\right] .
$$

where $w_{i}(t)$ - frequency distorting function which can be optimized to minimize the cross-correlations between users, and to minimize the off-peak auto-correlation of the ith line signal, $\phi_{i}(t)$ - information carrying phase component.

A base-band approach [5] follows from the fact that for the real spreading code $g_{i}(t)$, equation (1) can be rewritten in the exponential form as:

$$
s_{i}(t)=g_{i}(t) \cdot \operatorname{Re}\left\{e^{j \omega_{i} t} \exp \left[j \int_{0}^{t} w_{i}(\tau) d \tau\right] e^{\phi_{i}(t)}\right\} .
$$

The complex envelope $[1 \mid \sigma(t)$ of such a signal is given by:

$$
\sigma(t)=g_{i}(t) \exp \left[j \int_{0}^{t} w_{i}(\tau) d \tau\right] e^{\phi_{i}(t)}=\tilde{g}_{i}(t) e^{\phi_{i}(t)}
$$

Because signal is usually processed using digital signal processing (DSP) technology in the receiver, instead of the analogue spreading waveform:

$$
\tilde{g}_{i}(t)=g_{i}(t) \exp \left[j \int_{0}^{t} w_{i}(\tau) d \tau\right]
$$

one can use the polyphase complex spreading signature $\hat{g}_{i}(t)$. The length of the signature $\hat{g}_{i}(t)$ equals to the length of the original signature $g_{i}(t)$ multiplied by the number of samples per chip used in the receiver. and its pulse levels are weighted by the factor

$$
W_{i}(t)=\exp \left[j \int_{0}^{t} w_{i}(\tau) d \tau\right]
$$

Such an approach allows for the baseband correlational detection of the signals, easier optimization of the functions $w_{i}(t)$ for the given set of binary signatures, and facilitates implementation of the receivers.

In order to optimize the spreading sequences for the use in a 13 channel DS CDMA ATM WLAN. we have chosen a subset of 13 orthogonal Walsh functions [1] as a set of binary spreading codes $g_{i}(t), i=1,2, \ldots, 13$. These functions are listed in Table 1.

TABLE 1: SET OF 13 BINARY SPREADING SEQUENCES.

\begin{tabular}{|c|c|}
\hline Number & Binary spreading sequence \\
\hline 1 & $++++\cdots++++\cdots$ \\
\hline 2 & $++\cdots++++\cdots++$ \\
\hline 3 & $+++++\cdots++\cdots++$ \\
\hline 4 & $\cdots++\cdots++++\cdots++\cdots$ \\
\hline 5 & $++\cdots++\cdots++\cdots++$ \\
\hline 6 & $+\cdots++\cdots++\cdots++\cdots+$ \\
\hline 7 & $+++\cdots+++\cdots++\cdots+$ \\
\hline 8 & $+++\cdots+\cdots++\cdots+\cdots++$ \\
\hline 9 & $+\cdots+\cdots+\cdots+\cdots+\cdots+\cdots$ \\
\hline 10 & $+\cdots+\cdots+++\cdots+\cdots+++$ \\
\hline 11 & $++\cdots+\cdots++\cdots+\cdots+++$ \\
\hline 12 & $++\cdots++\cdots+++\cdots+\cdots$ \\
\hline 13 & $+\cdots+++++++++$ \\
\hline & \\
\hline
\end{tabular}

To minimize the cross-correlation between any pair of the spreading signatures, independently of the relative delay, we applied the described method with the functions $n_{i}(t), i=1,2, \ldots, 13$, being of the form:

$$
w_{i}(t)=2 \pi\left[\alpha_{i} \zeta(t / 16)+\beta_{i} \zeta(t / 8)+\gamma_{i} \zeta(t / 4)\right] .
$$

where the triangular wave $\zeta(t)$ is defined as:

$$
\zeta(t)=\sum_{n=-\infty}^{\infty} \lambda(t-n) .
$$

and

$$
\lambda(t)=\left\{\begin{array}{rc}
0, & t<0 \\
4 t-1 . & 0 \leq t<0.5 \\
-4 t+3, & 0.5 \leq t<1 \\
0, & t \geq 1
\end{array}\right.
$$

The obtained values of the parameters $\alpha_{i}, \beta_{i}, \gamma_{i}$. $i=1, \ldots, 13$ are given in Table 2 . The algorithm used to obtain them has been presented in [5].

\section{SIMULATION OF DS CDMA WATM LAN}

In order to simulate the 13 chamel DS CDMA WATM LAN we assumed the following: 
- a WATM cell consisted of 524 bits (424 bits of an ATM cell plus 100 bits of an overhead),

- BPSK was used as a modulation technique,

- the information contents of each WATM cell was random,

- relative delay between the signals corresponding to different channels was random with the delay step of 0.25 of a chip duration,

- the received power was equal for all of the channels.

The receiver antenna was simulated as a sum of the signals arriving from different channels, and the detection was a correlational one. The simulation was repeated for 2000 cells for each of the channels.

The obtained results show that the quality of transmission depends slightly on the signature used to spread the signal, so the achieved BER is not uniform for all of the channels, which is reflected by the number of errors occurring in the received WATM cells.

TABLE 2: SET OF THE OPTIMIZED COEFFICIENTS.

\begin{tabular}{|c|c|c|c|}
\hline Number & $\alpha$ & $\beta$ & $\gamma$ \\
\hline \hline 1 & -4.2315 & -2.1554 & -2.8487 \\
\hline 2 & -4.2040 & -2.0251 & -2.7369 \\
\hline 3 & -4.2127 & -2.0521 & -2.7685 \\
\hline 4 & -4.2597 & -2.0233 & -2.8507 \\
\hline 5 & -4.1610 & -2.1498 & -2.8281 \\
\hline 6 & -4.1504 & -2.1694 & -2.8278 \\
\hline 7 & -4.2512 & -2.1321 & -2.7522 \\
\hline 8 & -4.3205 & -2.0186 & -2.6968 \\
\hline 9 & -4.2259 & -2.0962 & -2.7669 \\
\hline 10 & -4.2763 & -2.0668 & -2.7818 \\
\hline 11 & -4.2678 & -2.1340 & -2.9848 \\
\hline 12 & -4.1794 & -2.1598 & -2.8193 \\
\hline 13 & -4.2054 & -2.0276 & -2.7752 \\
\hline
\end{tabular}

For all 13 chamnels, the obtained BER is equal to $2.6 \times 10^{-3}$. ranging from $1.3 \times 10^{-3}$ to $4.0 \times 10^{-3}$, and the histogram presenting the distribution of errors in the received WATM cells is presented in Figure 1.

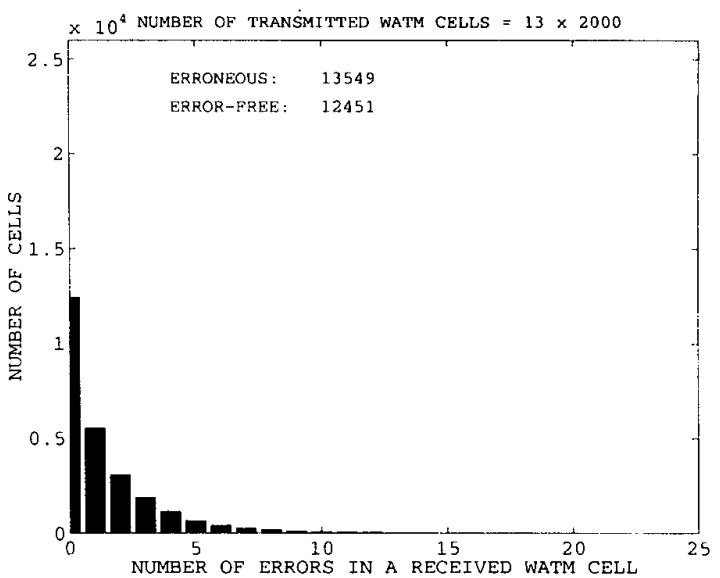

Figure 1: Histogram of the numbers of transmission errors in a received WATM cell for all 13 channels.

\section{Error Control with Hard Decision Decoding}

For the protection of ATM cells, we have designed an efficient coding scheme specifically for a WATM indoor environment [6]. The coding scheme is based on shortened $\mathrm{BCH}$ codes. The $\mathrm{BCH}$ code used for FEC adds 100 bits to each ATM cell. Results of throughput efficiency for hybrid Selective Repeat (SR) ARQ schemes on a channel with average bit error rate (BER) of $\bar{p}_{b}=1 \%$ are shown in Figure 2. As can be seen from the plots, a throughput of slightly less than $\eta=80 \%$ is obtained by introducing $m=100$ parity bits used to correct up to $t=10$ errors, where the number of information bytes can be chosen in the range of 50 to 70 bytes without significant impact on throughput.

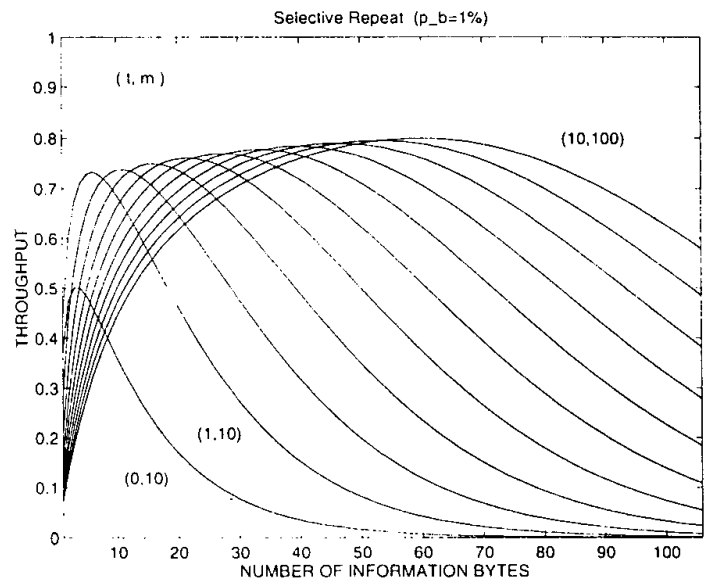

Figure 2: Throughput of a hybrid SR ARQ vs number of information bytes on a channel with bit error rate of $1 \%$ ( $t=$ errorcorrecting capability, $m=n u m b e r$ of parity bits). 


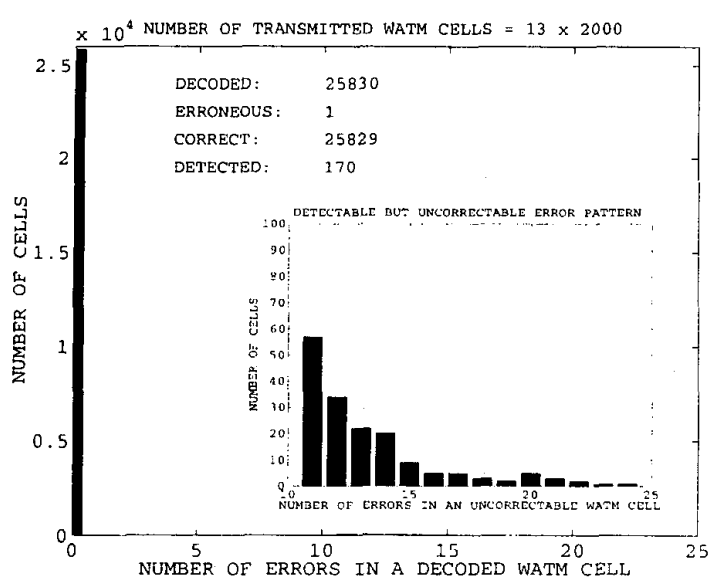

Figure 3: Histogram of the numbers of errors in a WATM cell after decoding for all 13 channels.

Using an error-correcting capability of $t=10$, we are able to correct the majority of those error events shown in Figure 1. The histogram of errors in a WATM cell after decoding and summation over all 13 channels is depicted in Figure 3. Out of the 26000 transmitted WATM cells, the $\mathrm{BCH}$ decoder is able to decode 25830 WATM cells whereas 170 WATM cells have been detected as being corrupted by an uncorrectable error pattern. Further on, a single WATM cell has been released erroneous with 19 bits in error and this gives a post-decoding BER of $1.4 \times 10^{-6}$. All other released WATM cells are error freè. For all 13 channels, the total number of cells which require retransmission is about $0.65 \%$, ranging from $0 \%$ to $2.15 \%$.

\section{ERRORS AND ERASURES DECODING}

To further improve the performance of the proposed DS CDMA system with error control, we now employ errors and erasures decoding instead of hard decision decoding. Because post-decoding BER performance is already sufficient with hard decision decoding, this approach is rather taken to reduce the number of retransmissions for real-time and delay-sensitive services.

Correction of errors and erasures has been implemented according to the algorithm described in [7] and was chosen for our application because of its simplicity. It uses a standard $\mathrm{BCH}$ decoding algorithm for a $t$ error-correcting $\mathrm{BCH}$ code and operates as-follows.

- Let the received word contain $e$ erasures.

- Replace the $e$ erased positions with zeros and decode this word with a standard $\mathrm{BCH}$ decoding algorithm.
- Replace the $e$ erased positions with ones and decode this word with a standard $\mathrm{BCH}$ decoding algorithm.

- Select that obtained codeword which relates to the smallest number of errors corrected outside the erased positions.

In order to identify whether a position in a codeword has to be considered as being erased, we have to define a threshold value for the correlator output of the receiver. As an example, Figure 4 shows relative frequency of normalized correlator output for CDMA channel 1, i.e. when the corresponding signature number 1 is used.

The quality of transmission depends slightly on the signature used to spread the signal. Accordingly, optimal thresholds are different for different signatures and the obtained results are listed in Table 3. A threshold of zero denotes simple hard decision decoding. Table 3 also shows the reduction of required retransmissions when hard decision is replaced by errors and erasures decoding. Actually, the total number of retransmissions, i.e. uncorrectable WATM cells, for all 13 channels is reduced by $28.8 \%$ from 170 to 121 WATM cells. In other words, only $0.46 \%$ of WATM cells would require a retransmission.

Figure 5 shows the histogram of errors in a WATM cell after correction of errors and erasures and summation over all 13 channels.

As the sub-figure indicates, the increase of correctly decoded WATM cells is achieved for those which are corrupted by 11 errors. Note also that all released WATM cells are now error free and the event of an erroneous decoding did not occur.

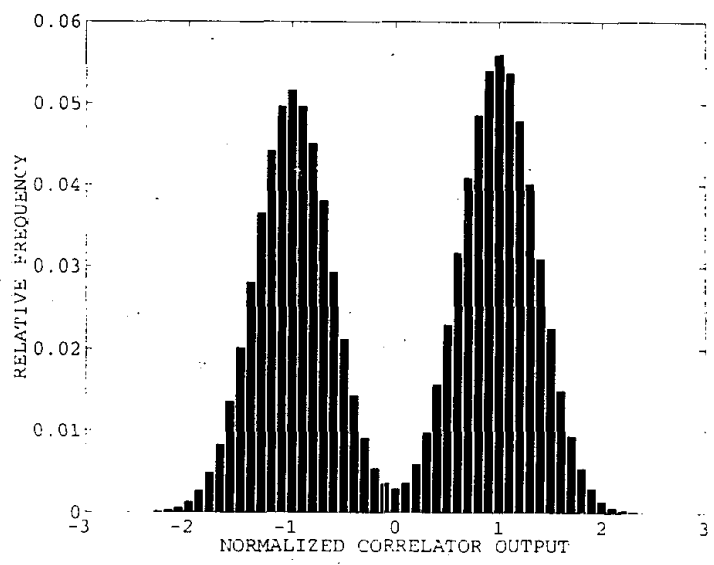

Figure 4: Histogram of the normalized correlator output for channel 1 and transmission of 2000) WATM cells. 
TABLE 3: NUMBER OF WATM CELLS WHICH REQUIRE RETRANSMISSION (ABSOLUTE AND IN \%)

\begin{tabular}{|c|c|c|c|}
\hline $\begin{array}{c}\text { CDMA } \\
\text { Channel }\end{array}$ & Hard & $\begin{array}{c}\text { Errors and } \\
\text { Erasures }\end{array}$ & Threshold \\
\hline \hline 1 & $42 / 2.1 \%$ & $28 / 1.4 \%$ & 0.070 \\
\hline 2 & $20 / 1 \%$ & $14 / 0.7 \%$ & 0.047 \\
\hline 3 & $43 / 2.15 \%$ & $37 / 1.85 \%$ & 0.026 \\
\hline 4 & $4 / 0.2 \%$ & $4 / 0.2 \%$ & 0 \\
\hline 5 & $2 / 0.1 \%$ & $2 / 0.1 \%$ & 0 \\
\hline 6 & $19 / 0.95 \%$ & $11 / 0.55 \%$ & 0.054 \\
\hline 7 & $20 / 1.0 \%$ & $12 / 0.6 \%$ & 0.068 \\
\hline 8 & $7 / 0.35 \%$ & $4 / 0.2 \%$ & 0.070 \\
\hline 9 & $3 / 0.15 \%$ & $3 / 0.15 \%$ & 0 \\
\hline 10 & $3 / 0.15 \%$ & $3 / 0.15 \%$ & 0 \\
\hline 11 & $0 / 0 \%$ & $0 / 0 \%$ & 0 \\
\hline 12 & $2 / 0.1 \%$ & $2 / 0.1 \%$ & 0 \\
\hline 13 & $5 / 0.25 \%$ & $1 / 0.05 \%$ & 0.037 \\
\hline TOTAL & $\mathbf{1 7 0 / 0 . 6 5 \%}$ & $\mathbf{1 2 1 / 0 . 4 6 \%}$ & \\
\hline & & & \\
\hline
\end{tabular}

\section{CONCLUSIONS}

In this paper we presented the simulation results for the 13 channel DS CDMA WATM LAN utilising optimized complex spreading signatures based on Walsh functions. The results are very promising, and indicate that with the application of a hybrid ARQ scheme with errors and erasures decoding, the number of WATM cells which would require retransmission is in the order of $0.46 \%$. Further research investigating the behaviour of the system without the perfect power control and incorporating the realistic indoor channel model is required.

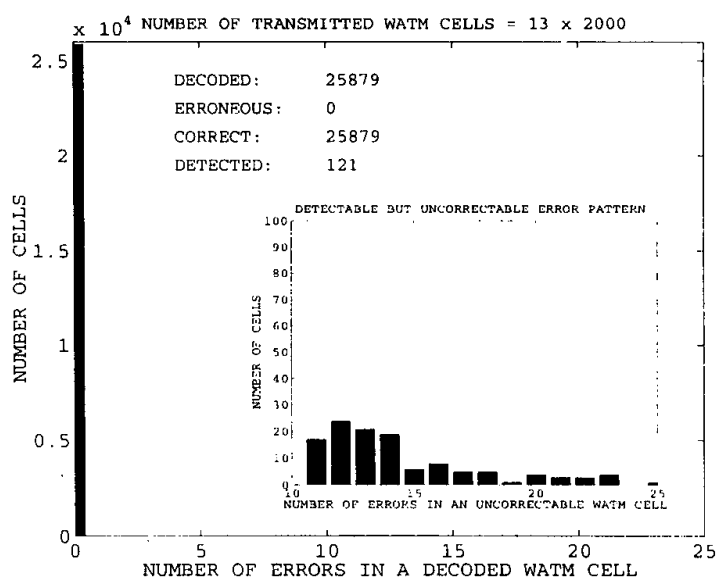

Figure 5: Histogram of the numbers of errors in a WATM cell after correction of errors and erasures for all 13 channels.

\section{REFERENCES}

[1] J.G.Proakis, Digital Communications. 3rd ed., New York: McGraw-Hill, 1995.

[2] TIA/EIA IS-95, "Mobile Station-Base Station Compatibility Standard for Dual-Mode Wideband Spread-Spectrum Cellular Systems", Telecom. Industry Assoc., July 1993.

[3] ETSI, "Agreement reached on radio interface for third generation mobile system, UMTS (Universal Mobile Telecommunications System)", ETSI, Press Release, Tdoc 40/98, Paris, France, 29 Jan. 1998.

[4] B.Wysocki and T.Wysocki, "A Method to Partially Suppress ISI and MAI for DS SS CDMA Wireless Networks", in Proc: ICC'97, Montreal, Canada, June 1997, pp.899-903.

15] B.J.Wysocki, T.A.Wysocki, and H.-J.Zepernick, "Error Performance of the 13-Channel DS CDMA WATM LAN", in Proc. ICUPC'98, Florence, Italy, Oct 1998, pp.19191923.

[6] H.-J.Zepernick, "Reliability, Throughput and Delay of Hybrid ARQ Schemes in a Wireless ATM LAN", RSL-TR007, ATRI, Perth, Australia, Dec. 1996.

17) G.C.Clark and J.B. Cain. Error-Correction Coding for Digital Communications, New York: Plenum Press, 1981. 